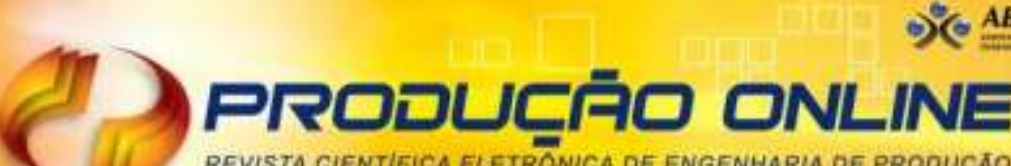 REVISTA CIENTIFICA ELETRONICA DE ENGENHARIA DE PRODUCATO ISSN 1676-1901
}

\section{CURVAS DE APRENDIZADO E FMEA NA ANÁLISE DE CONFIABILIDADE DO PROCESSO DE SEPARAÇÃO MANUAL DE UMA DISTRIBUIDORA DE MEDICAMENTOS}

\section{RELIABILITY ASSESSMENT OF A MANUAL-BASED PROCEDURE TOWARDS LEARNING CURVE MODELING AND FMEA ANALYSIS}

\author{
Gustavo Rech*E-mail: guugarech@gmail.com \\ Michel José Anzanello* E-mail: anzanello@producao.ufrgs.br \\ Camila Costa Dutra* E-mail: camila@producao.ufrgs.br \\ *Universidade Federal do Rio Grande do Sul (UFRGS), Porto Alegre, RS
}

\begin{abstract}
Resumo: $O$ processo de separação de medicamentos em centrais de distribuição (CD) é fundamentalmente embasado em atividades manuais, estando propenso à ocorrência de falhas como remessa de medicamentos trocados, quebrados ou vencidos ao cliente. Duas intervenções aparecem como promissoras para aprimorar a confiabilidade na operação de separação: (i) seleção e alocação de operadores adequados à operação, e (ii) análise de potenciais modos de falhas incorridas pelos operadores selecionados. Este artigo integra Curvas de Aprendizado (CA) e FMEA (Failure Mode and Effect Analysis) com vistas à redução da ocorrência de falhas no processo de separação manual de uma distribuidora de medicamentos. As CAs fornecem subsídios para a geração de um índice para identificação dos operadores mais propensos à realização das atividades. A FMEA é então aplicada ao processo de separação executado pelos operadores selecionados, visando identificar os principais modos de falha destes operadores. Para a caracterização da severidade das falhas na operação, o tradicional índice de severidade do FMEA é desdobrado em dois subíndices relacionados a aspectos financeiros e danos à imagem da empresa. Ao ser aplicado em uma CD de medicamentos, o método proposto reduziu significativamente a frequência e severidade de falhas no processo de separação.
\end{abstract}

Palavras-chave: Curvas de aprendizado. FMEA. Centros de distribuição. Medicamentos.

\begin{abstract}
Separation procedures in drug Distribution Centers (DC) are manual-based activities prone to failures such as shipping exchanged, expired or broken drugs to the customer. Two interventions seem as promising in improving the reliability in the separation procedure: (i) selection and allocation of appropriate operators to the procedure, and (ii) analysis of potential failure modes incurred by selected operators. This article integrates Learning Curves (LC) and FMEA (Failure Mode and Effect Analysis) aimed at reducing the occurrence of failures in the manual separation of a drug DC. LCs parameters enable generating an index to identify the recommended operators to perform the procedures. The FMEA is then applied to the separation procedure carried out by the selected operators in order to identify failure modes. It also deployed the traditional FMEA severity index into two sub-indexes related to financial issues and damage to company's image in order to characterize failures severity. When applied to a drug DC, the proposed method significantly reduced the frequency and severity of failures in the separation procedure.
\end{abstract}

Keywords: Learning Curves. FMEA. Distribution Centers. Drugs. 


\section{INTRODUÇÃO}

O aumento significativo da demanda em centros de distribuição (CD) de medicamentos tem impulsionado mudanças na organização e funcionamento destes centros. Apesar da elevada variedade de produtos distribuídos, a maioria enquadrase na classe de commodity e pode ser oferecido por qualquer outro CD. Essa situação faz com que os distribuidores não possuam exclusividade de clientes (OLIVEIRA; OLIVEIRA, 2004, 2005; SANTOS, 2006).

A concorrência entre CDs é focada em processos logísticos, fazendo com que poucas diferenças significativas sejam verificadas nos serviços prestados (OLIVEIRA; OLIVEIRA, 2004). A confiabilidade de sistemas de distribuição tem figurado como fator de diferenciação, conforme mencionado por Tontini e Sant'Ana (2008) e Grippa et al. (2005). Tais autores enumeram fatores capazes de comprometer a confiabilidade dos serviços em CDs, dentre os quais a grande variedade de produtos em circulação, perecibilidade de alguns itens e comportamento sazonal da demanda, entre outros.

A confiabilidade em CDs de medicamentos torna-se crucial por conta das diversas atividades envolvidas no processo: recepção, estocagem, separação, expedição e transporte (ATAMANCZUK et al., 2008). O processo de separação, fundamentalmente embasado em atividades manuais (ou com mínima intervenção de maquinário), é especialmente propenso à ocorrência de falhas, entre elas o envio de caixas de medicamentos com produtos faltantes ou quebrados aos clientes, remessa de itens vencidos, trocados ou abertos. Tais falhas comprometem não só a qualidade do serviço prestado e a imagem da empresa, como também podem conduzir a consequências sérias no caso de consumo de medicamentos vencidos/trocados pelo cliente. Por fim, a automação do processo de separação não figura como alternativa para a maioria dos CDs por conta de seus elevados custos de instalação e manutenção. De tal forma, duas intervenções aparecem como promissoras para assegurar sucesso na operação de separação: (i) seleção e alocação de operadores propensos à operação, e (ii) análise de potenciais modos de falhas incorridas pelos operadores selecionados.

Este artigo integra Curvas de Aprendizado (CA) e FMEA (Failure Mode and Effect Analysis) no processo de separação manual de uma distribuidora de medicamentos com vistas à redução da ocorrência de falhas. As CAs visam 
identificar os operadores mais propensos à realização das atividades. Tais operadores são definidos com base em um índice gerado a partir dos parâmetros oriundos da CA. A FMEA é então aplicada no processo de separação executado pelos operadores selecionados, visando identificar os principais modos de falha destes operários. Com vistas a caracterização da severidade das falhas identificadas, o tradicional índice de severidade do FMEA é desdobrado em dois subíndices abordando aspectos financeiros e danos à imagem da empresa por conta da ocorrência de falhas.

A metodologia proposta inova nos seguintes aspectos: (i) integra uma ferramenta clássica da área de produção, CA, a uma técnica consolidada para a identificação de modos de falha de processo, FMEA; (ii) gera um índice de adequação do operador à tarefa, com base nos parâmetros estimados pela CA, a partir do qual operadores são selecionados; e (iii) desdobra o índice de severidade da FMEA em dois subíndices específicos para medição da severidade dos prejuízos financeiros e danos à imagem da empresa por decorrência de erros na separação dos produtos.

Este artigo apresenta cinco seções, além da presente introdução. A Seção 2 apresenta breve referencial teórico em torno das CAs e FMEA. Na Seção 3 é descrita a metodologia proposta para redução de falhas no processo de separação. A Seção 4 apresenta os resultados da aplicação da metodologia em uma distribuidora de medicamentos. Uma conclusão encerra o artigo na Seção 5.

\section{REFERENCIAL TEÓRICO}

Fundamentos em torno das curvas de aprendizado e da técnica FMEA são apresentados nas seções seguintes.

\subsection{Curvas de Aprendizado (CAs)}

CAs são representações matemáticas do desempenho de um trabalhador quando submetido a uma tarefa manual repetitiva. Trabalhadores demandam menos tempo para executar a tarefa à medida que repetições são efetuadas, seja pela familiaridade adquirida com os meios de produção, adaptação às ferramentas 
utilizadas ou pela descoberta de atalhos para realização da tarefa (WRIGHT, 1936; TEPLITZ, 1991; JABER; GUIFFRIDA, 2007; ANZANELLO; FOGLIATTO, 2007a). Modelos de CAs vêm sendo propostos na literatura ao longo dos anos, com destaque para modelos de potência, como o de Wright (1936), e para modelos hiperbólicos.

O modelo de Wright é a função matemática de aprendizado mais conhecida na literatura, devido a sua simplicidade e eficiência na representação de dados empíricos. A expressão matemática do modelo é:

$$
t=U_{1} z^{b},
$$

na qual $z$ representa o número de unidades produzidas, $t$ designa o tempo (ou custo) médio acumulado para produção de $z$ unidades, $U_{1}$ é o tempo (ou custo) para produzir a primeira unidade, e $b$ corresponde à inclinação da curva $(-1 \leq b \leq 0)$.

O modelo hiperbólico de aprendizado permite uma descrição mais precisa do processo de aprendizado, se comparado ao modelo de Wright. O modelo hiperbólico de três parâmetros reportado por Mazur e Hastie (1978) é expresso pela equação:

$$
y=k(x+p) /(x+p+r),
$$

tal que $p+r>0$. Na Eq. (2), $y$ descreve o desempenho do trabalhador em termos de unidades produzidas após $x$ unidades de tempo de prática acumulada ( $y \geq 0$ e $x \geq 0), k$ corresponde ao limite superior de $y(k \geq 0)$ em unidades produzidas por tempo trabalhado, $p$ designa a experiência prévia na tarefa em unidades de tempo $(p \geq 0)$ medido em unidades produzidas, e $r$ é o tempo de operação demandado para atingir um nível $k / 2$, isto é, metade do desempenho máximo, também medido em número de unidades produzidas. O parâmetro $r$ é também interpretado como velocidade de adequação aos procedimentos demandados pela operação (UZUMERI; NEMBHARD, 1998).

O modelo hiperbólico oferece melhor caracterização do perfil de aprendizado, permitindo identificar as características de cada operador e, por consequência, aperfeiçoar a alocação de tarefas aos operadores (UZUMERI; NEMBHARD, 1998). Em Anzanello e Fogliatto (2007b), tarefas são alocadas a equipes de acordo com os parâmetros estimados pelo modelo hiperbólico de curva de aprendizado. Assim, equipes mais produtivas recebem tarefas mais longas, ao passo que as equipes caracterizadas por rápido aprendizado recebem tarefas com tamanhos menores de 
lote. O modelo hiperbólico também foi utilizado por Anzanello e Fogliatto (2010) com vistas à programação de tarefas caracterizadas por necessidade de aprendizado dos operadores.

\subsection{A Ferramenta FMEA}

A Análise do Modo e Efeito de Falha (FMEA - Failure Mode and Effect Analysis) é geralmente classificada em dois tipos, de produto e processo. A FMEA de processo é utilizada para analisar processos novos ou já existentes, visando aumentar a confiabilidade dos processos e reduzir os custos das ações de corretivas e de melhoria. Outros objetivos da FMEA incluem aumentar as atividades com foco no cliente, utilizar o conhecimento técnico de uma equipe multifuncional, dar suporte para a melhoria contínua, aperfeiçoar as lições aprendidas (por meio de documentação) e utilizar as melhores práticas da engenharia simultânea (BLUVBAND; GRABOV, 2009; YANG et al., 2006, CROWE; FEINBERG, 2001; GILCHRIST, 1993; LAFRAIA, 2001; SKELTON, 1997).

A FMEA normalmente é conduzida em duas fases. A primeira fase é relacionada à identificação dos possíveis modos de falha e seus efeitos. Isso inclui a coleta de informações funcionais dos componentes e processo alvo da análise. Ferramentas básicas tais como as sessões de brainstorming e diagramas de causaefeito, podem ser utilizadas para determinar a relação entre modos potenciais de falhas, seus efeitos e as causas relacionadas a esses modos de falhas para cada função analisada. Modos de falhas são eventos que provocam a diminuição parcial ou total de uma função do processo, afetando o seu desempenho (HELMAN; ANDERY, 1995). Segundo os mesmos autores, deve-se perguntar como o processo pode falhar, e não se falhará ou não. Na identificação dos efeitos de falha, é descrita a consequência do modo de falha percebida pelo cliente, ou seja, a partir do ponto de vista do consumidor, observa-se como o sistema é afetado. Enfatiza-se a possibilidade de ocorrência de mais de um efeito para cada modo de falha.

Com tais informações, se estima a severidade dos efeitos das falhas, isto é, avalia-se o quanto o respectivo efeito de falha pode prejudicar o cliente. Este indicador varia de 1 (efeito de menor importância) a 10 (cliente muito insatisfeito) conforme o grau de gravidade do problema (ver Quadro 1). Na sequência, são 
listadas as causas de falha, ou seja, eventos que provocam o aparecimento de um tipo de falha. Nesta etapa descreve-se de forma simples e concisa a causa fundamental da ocorrência do problema.

Quadro 1- Parâmetros para determinação do índice de severidade

\begin{tabular}{|c|c|c|}
\hline Índice & Severidade & Critério \\
\hline 1 & Nenhuma & Nenhum efeito perceptível. \\
\hline 2 & $\begin{array}{l}\text { Muito } \\
\text { pequena }\end{array}$ & Cliente mal percebe a falha. Efeito muito leve sobre o desempenho do sistema. \\
\hline 3 & Pequena & Cliente percebe a falha. Mínimo efeito sobre o desempenho do sistema. \\
\hline 4 & Mínima & Cliente começa a ficar irritado. Pequeno efeito sobre o desempenho do sistema. \\
\hline 5 & Moderada & Cliente um pouco insatisfeito. Efeito moderado sobre o desempenho do sistema. \\
\hline 6 & Significativa & $\begin{array}{l}\text { Desconforto do cliente. Performance degradada do produto ou sistema. Falha } \\
\text { parcial, mas operável. }\end{array}$ \\
\hline 7 & Alta & Cliente insatisfeito. Performance do produto ou sistema é gravemente afetada. \\
\hline 8 & Extrema & Cliente muito insatisfeito. Produto ou sistema inoperável, mas seguro. \\
\hline 9 & Grave & $\begin{array}{l}\text { Efeitos potenciais críticos. Possibilidade de danos físicos aos clientes e } \\
\text { complicações com regulamentações governamentais. }\end{array}$ \\
\hline 10 & Perigosa & $\begin{array}{l}\text { Efeitos críticos e repentinos. Relacionados com a segurança dos clientes (quando } \\
\text { há risco de morte) e não conformidade com regulamentações governamentais. }\end{array}$ \\
\hline
\end{tabular}

Fonte: adaptado de Stamatis (1995).

Logo após, são determinados os respectivos valores de ocorrência baseados na probabilidade de falha e na sua taxa em mil itens (ver Quadro 2). Para isto, utilizam-se dados de especificações de componentes e experiência anterior.

Quadro 2 - Parâmetros para determinação do índice de ocorrência

\begin{tabular}{|c|l|l|l|}
\hline Índice & \multicolumn{1}{|c|}{ Ocorrência } & \multicolumn{1}{|c|}{ Critério } & \multicolumn{1}{|c|}{ Proporção } \\
\hline 1 & Quase nunca & Insucesso improvável. Não há histórico de falhas & $1: 1.000 .000$ \\
2 & Muito remota & Falhas raras & $1: 20.000$ \\
3 & Remota & Suscetível a muito poucas falhas & $1: 4.000$ \\
4 & Muito baixa & Suscetível a poucas falhas & $1: 1000$ \\
5 & Baixa & Falhas ocasionais & $1: 400$ \\
6 & Moderada & Moderado número de falhas & $1: 80$ \\
7 & Moderadamente alta & Moderadamente elevado número de insucessos prováveis & $1: 40$ \\
8 & Alto & Alto número de falhas prováveis & $1: 20$ \\
9 & Muito alta & Muito alto o número de falhas prováveis & $1: 8$ \\
10 & Quase certa & Falhas quase certas. Histórico da existência de falhas em & $1: 2$ \\
\hline
\end{tabular}

Fonte: Adaptado de Stamatis (1995) e Capaldo, Guerrero e Rozenfeld (1999).

A atividade seguinte compreende a verificação dos controles atuais de prevenção e detecção de falhas e, a partir deles, definem-se valores para o índice de detecção. Este indicador é operacionalizado de forma similar aos dois índices anteriores: peso 10 para um problema de impossível percepção e peso 1 para um problema com detecção quase certa (ver Quadro 3) (GRUNSKE et al., 2007; SHARMA et al., 2005; PUENTE et al., 2002; STAMATIS, 1995; TENG; HO, 1996). 
Quadro 3 - Parâmetros para determinação do índice de detecção

\begin{tabular}{|c|l|l|}
\hline Índice & \multicolumn{1}{|c|}{ Detecção } & \multicolumn{1}{c|}{ Critério } \\
\hline 1 & Quase certa & Técnicas conhecidas e comprovadas disponíveis \\
2 & Muito alta & Análises de computador disponíveis no início do projeto \\
3 & Alta & Uso de simulação e/ou modelagem nas fases iniciais do projeto \\
4 & Moderadamente alta & Ensaios em protótipos nas fases iniciais \\
5 & Moderada & Ensaios nos itens antes da produção \\
6 & Baixa & Ensaios em itens similares \\
7 & Muito baixa & Ensaios no produto através de usando protótipos nas fases finais do \\
8 & Remota & projeto \\
9 & Muito remota & Apenas técnicas não confiáveis disponíveis \\
10 & Quase impossível & Nenhuma técnica conhecida disponível \\
\hline
\end{tabular}

Fonte: adaptado de Stamatis (1995).

A partir dos três índices (severidade, ocorrência e detecção), a FMEA ordena os modos de falha que geram os maiores riscos do problema chegar ao cliente através do RPN (Risk Priority Number) (FERNANDES; REBELATO, 2006). Com isso, é possível focar nos pontos que mais afetam a estratégia da empresa em relação ao consumidor. Um exemplo de formulário da ferramenta preenchido é mostrado na Figura 1. A última coluna desta figura representa o RPN, obtido pela multiplicação dos três índices determinados anteriormente. Este indicador hierarquiza as falhas de acordo com seus riscos.

Figura 1 - Exemplo de formulário de FMEA parcialmente preenchido

\begin{tabular}{|c|c|c|c|c|c|c|c|c|c|}
\hline \multirow[b]{2}{*}{ FMEA: POJETO/PROCESSO } & \multicolumn{9}{|c|}{ FMEA - ANÁLISE DOS MODOS DE FALHAS E SEUS EFEITOS } \\
\hline & \multicolumn{5}{|l|}{ ÁREAS ENVOLVIDAS: } & \multicolumn{4}{|l|}{ APROVAÇÁO DO CLIENTE: } \\
\hline PROCESSO/PRODUTO: & \multicolumn{9}{|l|}{ CLIENTE/PROJETO: } \\
\hline \multicolumn{10}{|c|}{\begin{tabular}{l|l} 
Recobrimento Superficial & \\
\end{tabular}} \\
\hline RESPONSÁVEL PROJETO/MANUFATURA: & \multicolumn{9}{|l|}{ EQUIPE: } \\
\hline $\begin{array}{l}\text { ITEM/NOMEIFUNÇÃO DO PROJETO/ } \\
\text { PROCESSO }\end{array}$ & $\begin{array}{l}\text { MODO DE FALHA } \\
\text { POTENCIAL }\end{array}$ & $\begin{array}{l}\text { EFEITO (S) DA FALHA EM } \\
\text { PODENCIAL }\end{array}$ & 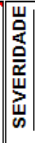 & $\begin{array}{l}\text { CAUSA (S) POTENCIAL DA } \\
\text { FALHA }\end{array}$ & 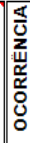 & $\begin{array}{l}\text { CONTROLE ATUAL DE } \\
\text { PREVENÇÄO }\end{array}$ & $\begin{array}{c}\text { CONTROLE ATUAL DE } \\
\text { DETECÇÄO }\end{array}$ & 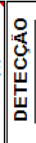 & 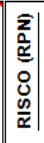 \\
\hline \multirow[t]{3}{*}{$\begin{array}{l}\text { Revestimento Protetor: } 0,1 \mathrm{~mm} \text { de Cromo em } \\
\text { Aço Carbono }\end{array}$} & \multirow[t]{3}{*}{$\begin{array}{l}\text { Composição inadequada } \\
\text { do banho }\end{array}$} & Recobrimento inadequado & 5 & $\begin{array}{l}\text { Presença de particulas } \\
\text { contaminates }\end{array}$ & 7 & & & 9 & 315 \\
\hline & & Porosidades & 8 & Mistura inadequada & 6 & & & 5 & 240 \\
\hline & & $\begin{array}{l}\text { Acabamento superficial } \\
\text { inadequado }\end{array}$ & 6 & Condensação inadequada & 7 & & & 4 & 168 \\
\hline
\end{tabular}

Fonte: Adaptado de Helman e Andery (1995)

A segunda fase é realizada quando o valor do RPN ultrapassa o valor desejado e, por isso, ações corretivas ou alterações de projeto são requeridas. Com o intuito de definir itens que requerem planos de ações, alguns critérios devem ser 
adotados, definidos a partir do agrupamento de todas as causas de falha do sistema da seguinte maneira (STAMATIS, 1995): (i) risco associado maior ou igual a 120; ou (ii) severidade alta à perigosa (maior ou igual a 7); ou (iii) ocorrência moderada à quase certa (maior ou igual a 6); ou (iv) probabilidade de detecção muito baixa à quase impossível (maior ou igual a 7).

As ações corretivas visam à diminuição da probabilidade de não detecção do modo de falha, enquanto as alterações no projeto buscam reduzir a severidade das falhas e a probabilidade de sua ocorrência, sendo esta alternativa utilizada para modos de falha que possuem um alto risco associado a sua ocorrência. $\mathrm{Na}$ sequência, realiza-se nova análise dos modos e efeitos de falha, a fim de verificar eventuais reduções no RPN. Ao final do processo, um relatório deve ser gerado e as modificações requeridas devem estar completas, de modo a minimizar o número de modos potenciais de falhas (TENG; HO, 1996).

É importante enfatizar que o mapeamento de falhas por meio da FMEA requer um trabalho em equipe com foco em fatores como know-how, controle e tempo de preenchimento para a identificação dos principais pontos do processo (ALMEIDA, 2006; POSSO, 2009). Assim, a formação de uma equipe multidisciplinar torna a aplicação mais completa e precisa. Posso (2009) alerta para a utilização de aspectos subjetivos na aplicação do método, os quais podem gerar diferentes resultados e interpretações. Em contrapartida, o trabalho em equipe possibilita ganhos motivacionais e comportamentais constatados por Roos et al. (2008). O autor destaca o aumento do comprometimento das pessoas e dos valores em prol de um avanço da empresa e do próprio funcionário.

\section{MÉTODO}

O método proposto visa minimizar falhas no processo de separação de uma distribuidora de medicamentos. Processos de separação neste segmento são fundamentalmente embasados em atividades manuais, fazendo com que a eficiência do processo em termos de velocidade e confiabilidade seja dependente da adequação dos operadores aos preceitos de execução da operação.

O método sugerido é operacionalizado em dois passos. O Passo 1 inicia com a seleção do grupo de produtos com maior representatividade, visto que o processo 
analisado apresenta elevada diversidade de medicamentos. Na sequência, propõese a modelagem por CAs para identificar os operadores mais propensos à realização das atividades. Tais operadores são definidos com base em um índice gerado a partir dos parâmetros oriundos da CA. O Passo 2 aplica a ferramenta FMEA no processo de separação executado pelos melhores operadores oriundos do Passo 1, visando identificar os principais modos de falha destes operadores; na sequência são elencadas proposições de melhoria para aumentar a confiabilidade do processo de separação. Os passos são detalhados nas seções seguintes.

\subsection{Passo 1: Seleção dos operadores mais adequados à execução dos procedimentos de separação}

Selecionam-se inicialmente os produtos mais relevantes em termos financeiros no setor de separação manual da empresa. Esta seleção utiliza a técnica $A B C$, a qual se baseia nas "movimentações de valor" valendo-se da demanda e do valor individual de cada item (SLACK, 2007). A metodologia proposta é aplicada no conjunto de itens tido como Classe $\mathrm{A}$, reduzindo a coleta de dados associados a itens de menor valor. Apesar de a classificação $A B C$ reduzir significativamente o número de itens analisados, a grande variedade de características dos produtos e seus diversos destinos demandam permanente readequação dos operadores aos preceitos de realização das operações. Essa situação mostra-se adequada à modelagem por CA.

Na sequência, coletam-se dados de desempenho dos operadores candidatos a executarem a operação. Os dados devem ser coletados no início da operação, sendo armazenados até que não sejam percebidas alterações significativas no padrão de desempenho. A coleta pode ser efetivada pela contagem do número de itens separados em determinado intervalo de operação. Os dados devem ser coletados para cada operador, de forma a gerar parâmetros característicos para cada candidato.

A modelagem dos dados de desempenho utiliza a curva hiperbólica de três parâmetros, apresentada na Eq. (2). Os parâmetros da curva hiperbólica podem ser estimados pelas rotinas de regressão não-linear disponíveis em aplicativos de análise estatística (neste estudo foi utilizado o software Matlab). Dados de 
desempenho são modelados como uma variável y (número de unidades separadas), a qual é dependente do tempo de processamento $x$. O modelo hiperbólico é utilizado devido ao seu desempenho satisfatório em estudos empíricos, porém outros modelos de curvas de aprendizado podem ser testados (NEMBHARD; UZUMERI, 2000 e ANZANELLO; FOGLIATTO, 2007b, 2010).

A definição dos operadores mais adequados à realização dos procedimentos de separação apóia-se em critérios de (i) experiência prévia na atividade, quantificada pelo parâmetro $p$, e (ii) velocidade de adequação aos procedimentos demandados, quantificada pelo parâmetro $r$. Os critérios (i) e (ii) são assumidos como suficientes na identificação dos operadores mais confiáveis e menos propensos à execução de erros. Os parâmetros $p$ e $r$ são então consolidados em um índice adimensional pela Equação (3).

$$
A_{i}=\frac{p_{i}}{r_{i}}
$$

na qual, $i(i=1, \ldots, l)$ denota os operadores analisados e $A$ é o coeficiente de adequação do operador $i$ à operação analisada. Os operadores responsáveis por elevados valores de $A$ são selecionados. Essa lógica de seleção encontra sustentação na natureza dos parâmetros $p$ e $r$ : Elevados valores de $p$ são desejados, visto que denotam operadores com elevada experiência e menor propensão a erros; e reduzidos valores de $r$ são preferidos, visto que denotam operadores com rápida adequação aos procedimentos, o que nesse estudo é assumido como clareza na execução dos mesmos e que acaba por traduzir-se em menor probabilidade de erros.

É importante enfatizar que a inclusão de um novo operador na análise demanda nova coleta de dados de desempenho e nova modelagem por CA.

\subsection{Passo 2: Aplicação da FMEA à operação de separação executada pelos operadores selecionados}

Este passo utiliza a ferramenta FMEA para identificar os pontos críticos do processo executado pelos operadores selecionados no Passo 1. Assume-se que a aplicação da FMEA em um processo executado por operadores com maior 
experiência prévia e rápido aprendizado pode ser beneficiada pela menor probabilidade de ocorrência de falhas.

Este trabalho propõe a divisão do índice de severidade em dois subíndices: severidade financeira dos custos incorridos por erros de separação e danos à imagem da empresa por conta de tais erros. Com isso, o cálculo do RPN é composto por quatro componentes: (i) severidade financeira $(S F)$, (ii) severidade de danos à imagem da empresa $(D l)$, (iii) frequência de ocorrência $(F)$, e (iv) detecção atual $(D)$, conforme apresentado na equação (4). Repare que os índices relativos à severidade são calculados pela raiz quadrada dos valores, de forma a manter o valor máximo de $\mathrm{RPN}=1000$. É importante enfatizar que as alterações propostas na geração do RPN modificam aspectos consolidados da FMEA, como a tradicional seleção dos modos de falha com RPN superior a 120. Também é fundamental a seleção de especialistas confiáveis para o preenchimento dos novos índices propostos, sob pena de distorção das escalas dos índices.

$$
R P N=\sqrt{S F} \times \sqrt{D I} \times F \times D
$$

O índice SF foca-se nas perdas diretas para a empresa, ou seja, aquelas que demandam recursos para serem contornados, como quebras e trocas. Dados históricos destes custos devem ser levantados para a operação em análise, e então vinculados à escala de 1 a 10 com o auxílio de especialistas de processo. O valor 10 denota o maior custo oriundo de um erro de separação, e deve ser estimado em bancos históricos contendo custos de ressarcimento a clientes ou prejuízo com quebras/trocas, entre outros.

O índice de severidade dos danos à imagem da empresa $(D I)$ está associado a prejuízos na relação do cliente com a organização e, por ser subjetivo, é de mais complexa obtenção. Este estudo sugere vincular "Natureza dos danos" decorrentes de erros de separação a uma escala de 1 a 10 (onde 10 denota risco à saúde do cliente pelo consumo de produto trocado/vencido). Tais índices são levantados por especialistas de processo. As relações sugeridas são apresentadas no Quadro 4. 
Quadro 4- Escala sugerida para os danos à imagem $(D I)$ da empresa

\begin{tabular}{|l|c|}
\hline \multicolumn{1}{|c|}{ Natureza dos danos } & $\begin{array}{c}\text { Índice de severidade dos danos } \\
\text { à imagem da empresa }\end{array}$ \\
\hline Risco à saúde do cliente & 10 \\
Perda do cliente & 9 \\
Qualidade do serviço e do produto & 8 \\
comprometidos & 7 \\
Cliente parcialmente insatisfeito & 6 \\
Problema não percebido pelo cliente ou & 5 \\
irrelevante & 4 \\
\hline
\end{tabular}

Os demais índices da FMEA, associados à frequência de ocorrência e potencial de detecção, permanecem inalterados.

\section{RESULTADOS E DISCUSSÕES}

O CD analisado responde por $60 \%$ da distribuição de medicamentos no Estado do Rio Grande do Sul, entre filiais da rede e outras farmácias. O processo de separação manual de itens apresenta problemas evidenciados por reclamações de clientes e indicadores internos. A identificação dos operadores mais propensos à execução da tarefa de separação, bem como uma análise detalhada dos potenciais modos de falha destes operadores, aparece como sistemática promissora para reduzir erros de separação.

Selecionaram-se 250 itens (classe A) considerados primordiais para o faturamento da empresa. Apesar do elevado número de produtos selecionados, o procedimento diminuiu significativamente a tarefa de coleta de informações.

Dados de desempenho de 5 operadores executando os procedimentos de separação dos itens selecionados foram coletados no início de cada turno. Para tanto, contabilizou-se o número de unidades separadas a cada intervalo de 30 segundos de operação. Os produtos separados apresentavam características físicas (dimensões, formatos e pesos) semelhantes, de permite a demandar esforços homogêneos para execução dos procedimentos. Os dados são apresentados na Tabela 1. O aumento no número de unidades separadas denota maior adequação do operador à tarefa com a evolução do tempo de operação. 
Tabela 1 - Número de unidades separadas por operador

\begin{tabular}{cccccc}
\hline $\begin{array}{c}\text { Intervalo de } \\
\text { operação }\end{array}$ & Operador $\mathbf{1}$ & Operador $\mathbf{2}$ & Operador $\mathbf{3}$ & Operador $\mathbf{4}$ & Operador $\mathbf{5}$ \\
\hline $00: 30$ & 9 & 8 & 4 & 10 & 6 \\
$01: 00$ & 8 & 11 & 5 & 9 & 6 \\
$01: 30$ & 11 & 16 & 4 & 11 & 5 \\
$02: 00$ & 13 & 12 & 4 & 14 & 7 \\
$02: 30$ & 11 & 21 & 7 & 16 & 6 \\
$03: 00$ & 12 & 15 & 8 & 15 & 8 \\
$03: 30$ & 9 & 17 & 5 & 16 & 9 \\
$04: 00$ & 13 & 16 & 6 & 10 & 8 \\
$04: 30$ & 11 & 18 & 9 & 13 & 7 \\
$05: 00$ & 11 & 15 & 7 & 14 & 10 \\
\hline
\end{tabular}

O modelo hiperbólico na Eq. (2) é utilizado para ajustar os dados da Tabela 2 , sendo o número de unidades modelado como variável y e o intervalo de produção como variável $x$. Um exemplo de curva de aprendizado hiperbólica de 3 parâmetros gerada para o operador 3 é apresentado na Figura 2.

Figura 2 - Curva de aprendizado para o operador 3

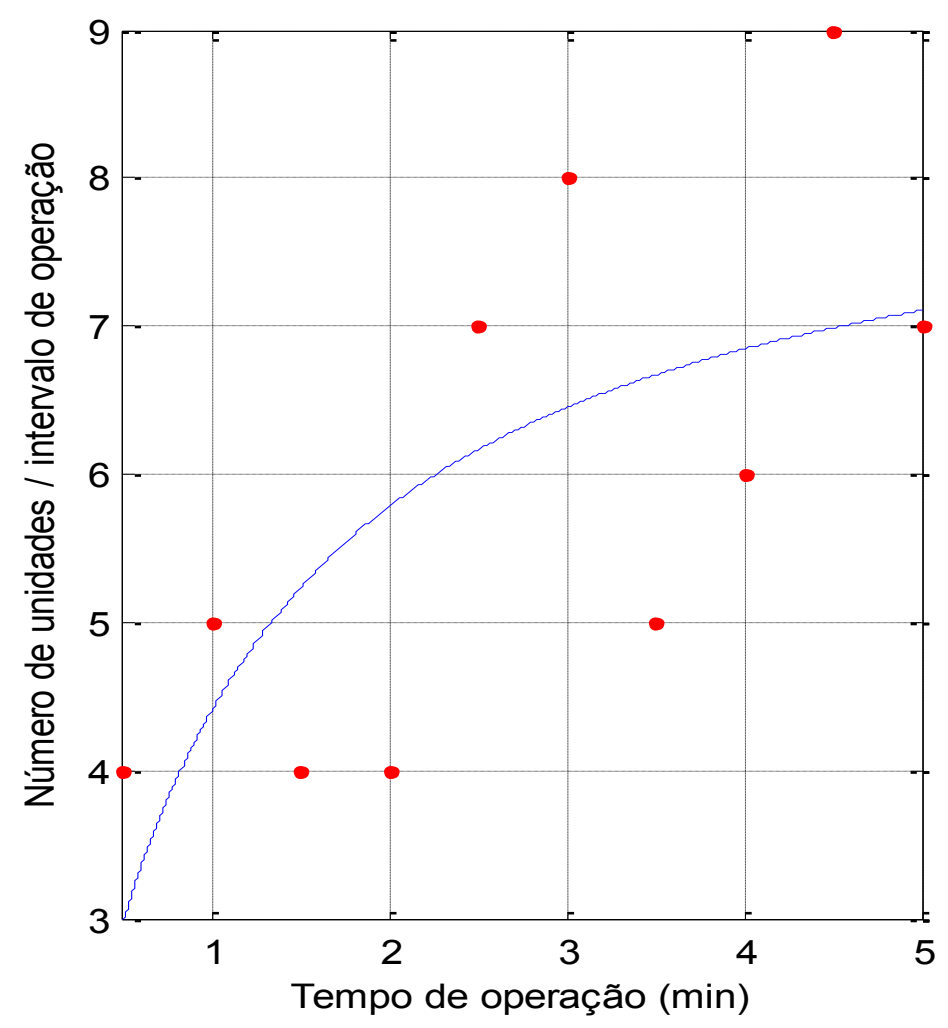

Os parâmetros resultantes da modelagem, bem como o índice $A$ gerado pela Eq. (3), são apresentados na Tabela 2. 
Tabela 2 - Coeficientes $A$ dos operadores avaliados

\begin{tabular}{ccccc}
\hline Operador & $p$ (número de unidades) & $r$ (número de unidades) & $\mathrm{A}$ & $\mathrm{R}^{2}$ \\
\hline 1 & 7,97 & 14,79 & 0,54 & 0,78 \\
2 & 14,15 & 21,64 & 0,65 & 0,91 \\
3 & 8,13 & 37,81 & 0,22 & 0,71 \\
4 & 8,42 & 25,33 & 0,33 & 0,85 \\
5 & 13,69 & 39,51 & 0,35 & 0,84 \\
\hline
\end{tabular}

A Tabela 2 aponta os operadores 2 e 1 como os mais aptos a realizarem a atividade de separação, por conta de seus elevados coeficientes $A$. Ao analisar os parâmetros $p$ e $r$, percebe-se que o operador 1 é caracterizado por uma reduzida experiência prévia (baixo $p$ ), porém o mesmo apresenta rápida assimilação dos preceitos da tarefa de separação (baixo r); o operador 2, por sua vez, apresenta elevada experiência anterior (elevado p). A seleção de dois operadores para execução da tarefa de separação permite o rodízio dos trabalhadores, e consequente redução de problemas ergonômicos decorrentes de esforços repetitivos.

Na sequência, identificaram-se os modos de falha para os operadores 1 e 2 por meio da FMEA de processo. Os possíveis modos de falha do processo referemse à falta, sobra ou troca de itens na embalagem, quebra durante separação, e remessa de itens vencidos, conforme apresentado na Figura 3. O preenchimento dos campos da FMEA seguiu as etapas descritas na Seção 2.2. Helman e Andery (1995) ressaltam que ações de melhoria devem focar-se em modos de falha com índices RPN maiores do que 120, fazendo com que ações preventivas sejam implementadas para os três modos de falha preponderantes da Figura 3: (i) item trocado, (ii) item quebrado, e (iii) item vencido. Tais melhorias, bem como os resultados decorrentes da sua implementação, são apresentadas na sequência.

(i) Item trocado: compreende os produtos que são trocados entre as caixas de separação (volumes de acondicionamento dos itens que chegam ao cliente final), causando problemas financeiros e de imagem à empresa. Como forma de prevenção, desenvolveu-se um marcador colorido anexado à caixa em separação; tal marcador evita que ocorram trocas entre os volumes manuseados por diferentes operadores executando a mesma atividade. Além disso, implantou-se uma política de revisão no 
endereçamento dos produtos e afastamento de itens semelhantes que potencialmente poderiam conduzir a equívocos operacionais.

(ii) Item quebrado: produtos geralmente são quebrados por conta de seu manuseio inadequado. Para tanto, implementou-se uma sistemática contínua de treinamento dos operadores com vistas à padronização da atividade de separação. Foram eliminados movimentos desnecessários no procedimento, além de intenso monitoramento de descuidos que pudessem danificar os itens. Por fim, passou a utilizar proteção extra no interior da caixa, como sacos de ar e revestimento de isopor.

(iii) Item vencido: tal falha foi contornada pela formulação de uma sistemática de inspeção contínua executada pelo responsável por estoques e pelo operador de separação. Tais profissionais somente estão autorizados a liberar e separar pedidos após conferência da data de validade do medicamento. Outra melhoria refere-se ao posicionamento correto dos medicamentos na esteira, de maneira a facilitar a visualização da data de validade gravada na embalagem.

As melhorias implementadas foram monitoradas por 45 dias, reduzindo significativamente 0 índice de ocorrência dos três modos de falha, conforme atestado pelos novos valores de RPN na Figura 3. Também é possível constatar que o controle de detecção melhorou devido ao treinamento fornecido aos operadores. 
Figura 3 - FMEA do processo de separação

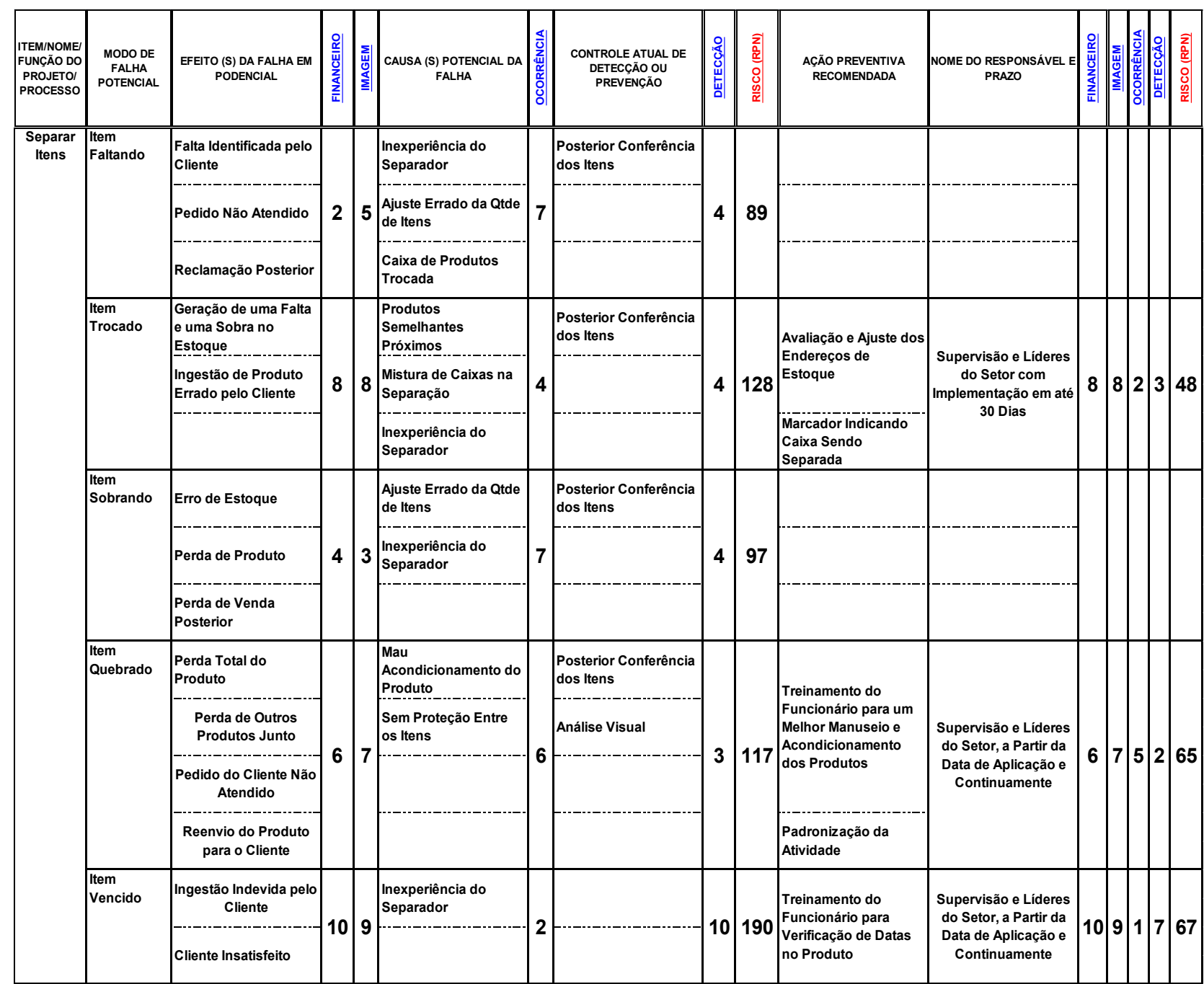

\section{CONCLUSÃO}

Processos de separação embasados em atividades manuais são propensos à ocorrência de falhas por conta de descuidos inerentes à atividade humana e deficiência de procedimentos padronizados. Em CD de medicamentos, tais deficiências acarretam na remessa de medicamentos trocados, quebrados ou vencidos ao cliente, comprometendo aspectos financeiros e de imagem da empresa.

Este artigo apresentou uma sistemática baseada na integração de CA e FMEA para reduzir a incidência de falhas no processo de separação manual de uma CD de medicamentos. Para tanto, dados de desempenho dos operadores candidatos à execução da separação foram modelados por meio das CAs, gerando um índice de adaptação dos operadores ao procedimento. Os operadores 
selecionados foram analisados pela técnica FMEA, com vistas à identificação dos principais modos de falha destes operadores. No método proposto, o tradicional índice de severidade do FMEA foi desdobrado em dois subíndices relacionados a aspectos financeiros e danos à imagem da empresa. Por fim, as ações de melhorias decorrentes da FMEA foram aplicadas com sucesso no processo de separação de uma CD de medicamentos, reduzindo significativamente a frequência e severidade de falhas no processo.

Futuros desdobramentos incluem o desenvolvimento de métodos alternativos para a seleção dos operadores mais propensos à execução das atividades, integrando métodos numéricos aos parâmetros oriundos das CAs. Também avaliada a utilização de ferramentas associadas aos princípios de Produção Enxuta para aprimoramento dos procedimentos executados pelos operadores selecionados e redução de perdas produtivas.

\section{REFERÊNCIAS}

ALMEIDA D.A. et al. Gestão do conhecimento na análise de falhas: mapeamento de falhas através de sistema de informação. Revista Produção, v. 16, n. 1, p. 171-188, 2006.

ANZANELLO M. J.; FOGLIATTO F. S. Curvas de aprendizado: estado da arte e perspectivas de pesquisa. Revista Gestão e Produção, São Carlos, v. 14, n. 1, p. 109-123, Jan/Abr 2007a.

ANZANELLO M. J.; FOGLIATTO F. S. Learning curve modelling of work assignment in mass customized assembly lines. International Journal of Production Research, v. 45, n. 13, p. 2919-2938, 2007b.

ANZANELLO, M. J.; FOGLIATTO, F. Scheduling learning dependent jobs in customized assembly lines. International Journal of Production Research, v. 48, p. 6683-6699, 2010.

ATAMANCZUK, M. J. ; FILHO, J. A. R.; PIETROBON, F. Gerenciamento de armazém através da ferramenta FMEA: um estudo de caso para o ramo supermercadista. In: IV Encontro de Engenharia e Tecnologia dos Campos Gerais, Paraná, 2008. Anais...Paraná, 2008.

BLUVBAND, Z.; GRABOV, P. Failure Analysis of FMEA. IEEE Transactions on Reliability, 2009. 
CAPALDO, D.; GUERRERO, V.; ROZENFELD, H. FMEA (failure mode and effect analysis). 1999. Disponível em: < http://www.numa.org.br >. Acesso em: nov. 2010.

CROWE, D.; FEINBERG, A. Design for Reliability., Boca Raton: CRC Press LLC, 2001.

FERNANDES, J. M. R.; REBELATO, M. G. Proposta de um Método para Integração entre QFD e FMEA. Revista Gestão \& Produção, v.13, n. 2, p.245-259, 2006.

FOGLIATTO, F.; RIBEIRO, J. L. Confiabilidade e manutenção industrial. Rio de Janeiro: Elsevier, 2009.

GILCHRIST, W. Modelling failure modes and effects analysis. International Journal of Quality \& Reliability Management, v. 10, n. 5, p. 16-23, 1993.

GRIPPA, D. B.; LEMOS, F. O.; FOGLIATTO, F. S. Analogia e combinação de previsões aplicados à demanda de novos produtos. In: ENCONTRO NACIONAL DE ENGENHARIA DE PRODUÇÃO, 25,. 2005. Anais...RS, 2005.

GRUNSKE, L.; COLVIN, R.; WINTER, K. Probabilistic Model-Checking Support for FMEA. In Proc. 4th INTERNATIONAL CONFERENCE ON THE QUANTITATIVE EVALUATION OF SYSTEMS, QEST 07. IEEE Computer Society, 2007. Anais...p. 119-128, 2007.

HELMAM, H.; ANDERY P. R. P. Análise de falhas (aplicação dos métodos de fmea - fta). Belo Horizonte: Fundação Christiano Ottoni, Escola de Engenharia da UFMG, p. 2-4, 1995.

JABER, M.; GUIFFRIDA, A. Observations on the economic order (manufacture) quantity model with learning and forgetting. International Transactions in Operational Research, v.14, n.2, p.91-104, 2007.

LAFRAIA, J. R. B. Manual de confiabilidade, mantenabilidade e disponibilidade. Rio de Janeiro: Qualitymark, 2001. 374 p.

MAZUR, J. E.; HASTIE, R. Learning as Accumulation: a reexamination of the learning curve. Psychological Bulletin, Washington DC, v. 85, n. 6, p. 1256-1274, 1978.

NEMBHARD, D. A.; UZUMERI, M. V. An individual-based description of learning within an organization. IEEE Transactions on Engineering Management, New Jersey, v. 47, n. 3, p. 370-378, 2000.

OLIVEIRA, J. L. R.; OLIVEIRA S. L. Análise de clientes de uma distribuidora de medicamentos. Revista Contemporânea de Economia e Gestão, v. 02, n. 2, p. 4855, 2004. 
OLIVEIRA, J. L. R.; OLIVEIRA S. L. Logística de distribuição e controle de estoques em uma distribuidora de medicamentos. Revista Produção Online, v. 05, n. 3, 2005.

POSSO R.; ESTORILIO C. Identificação dos fatores de influência na aplicação do método failure mode and effect analysis- FMEA de processo: um estudo em produtos estampados. Revista Produto \& Produção, v. 10, n. 2, p.87 - 107, 2009.

PUENTE, J. et al.. A decision support system for applying failure mode and effects analysis. International Journal of Quality \& Reliability Management, v. 19, n. 2, p. 73-83, 2002.

ROOS, C.; MORAES, J. A. R.; ROSA, L. C. Melhoria da qualidade nos serviços de transporte utilizando a ferramenta FMEA. Revista Gestão Industrial, v. 04, n. 01, p. 148-159, 2008.

SANTOS, A. centros de distribuição como vantagem competitiva. Revista de Ciências Gerenciais, 2006.

SHARMA, R.K.; KUMAR, D.; KUMAR, P. Systematic failure mode effect analysis (FMEA) using fuzzy linguistic modeling. International Journal of Quality \& Reliability Management, v. 22, n. 9, p. 986-1004, 2005.

SKELTON, B. Process safety analysis: an introduction. Warwickshire: Institution of Chemical Engineers, 1997. 220 p.

SLACK, N.; CHAMBER, S.; JOHNSTON, R. Administração da produção. São Paulo: Atlas, 2007.

STAMATIS, D. H. Failure mode and effect analysis: FMEA from theory to execution. Milwaukee, US, American Society for Quality, 2003.

TENG, S. H.; HO, S. Y. Failure mode and effects analysis: an integrate approach for product design and process control. International Journal of Quality \& Reliability Management, v. 13, n. 5, p. 8-26, 1996.

TEPLITZ, C. J. The learning curve deskbook: A reference guide to theory, calculations and applications. Quorum Books: New York, 1991.

TONTINI, G.; SANT'ANA A. J. Interação de atributos atrativos e obrigatórios de um serviço na satisfação do cliente. revista Produção, v. 18, n. 1, p. 112-125, 2008.

UZUMERI, M.; NEMBHARD, D. A population of learners: a new way to measure organizational learning. Journal of Operations Management, v. 16, p. 515-528, 1998.

WRIGHT, T. Factors affecting the cost of airplanes. Journal of the Aeronautical Sciences, v. 3, n.2, p. 122-128, 1936. 
YANG, C. et al. A study on applying FMEA to improving ERP introduction: an example of semiconductor related industries in Taiwan. International Journal of Quality \& Reliability Managemen, v. 23, n. 3, p. 298-322, 2006.

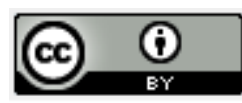

Artigo recebido em 08/08/2011 e aceito para publicação em 07/08/2012. 